\title{
Using drift diffusion model to understand age-related differences in inhibitory control
}

\author{
A.C. Tsai*, S. Nayak, C.-C. Hsu, C.-S. Kuo, S.-H. Li, J.O.S. Goh, Y.-P. Chao, \\ L. Jingling, S.-L. Yeh \\ NeuroImaging group at the Institute of Statistical Science, Academia Sinica, Taipei, Taiwan \\ *e-mail:arthur@stat.sinica.edu.tw
}

Key words: emotional inhibition, emotional stop signal paradigm, drift-diffusion model, ICA, fMRI

Many situations require one to inhibit the impulse to react to others' emotional facial displays. Considerable evidence, however, points to age-related changes in response inhibition in older adults. In addition, evidence also shows more automatic regulation of affect in older compared to younger adults. How these two opposing processes operate in young and older adults remains unclear. In this study, a drift diffusion model was used to each participant's behavioral data to extract components of psychological processing, including measures of caution, motor execution time, and stimulus processing speed. The individual differences in the resulting components were further examined in relation to fMRI activation to evaluate the underlying neural correlates and the emotional modulation in young and older adults. Thirty-two young and 32 older normal adults underwent an emotional stop signal paradigm (ESSP) in an fMRI experiment with disgusted and neutral emotional faces. Participants were instructed to make a target response (Go) as quickly as possible to face stimuli unless a red border appeared (between 40 to $400 \mathrm{~ms}$ of face onset), in which case they were to withhold their response (Stop). Young adults made more Stop than Go errors to neutral faces. Disgusted faces increased Stop false alarms, decreased Go hits, and also reduced reaction times compared to neutral faces. Older adults made more Go than Stop errors to neutral faces. Disgusted faces increased Stop false alarms, increased Go Hits, and also reduced reaction times compared to neutral faces. Two-sample t-tests are used to delineate the role of aging in emotional inhibition tasks. The results show that older adults tend to engage more neural circuits than young adults including striatum and hippocampus regions. Younger adults show more focal activations in right inferior frontal cortex, inferior parietal lobule and postcentral gyrus for inhibition contrasts. Using drift-diffusion modeling principles, our findings suggest whereas negative affect increases the rate of evidence accumulation for target and stop feature decisions in younger adults, negative affect lowers the decision criterion in older adults. 